\title{
List of reviewers
}

JOURNAL OF ADVANCES IN MATHEMATICS (2018) Vol 14.

The editorial team of the journal would like to thank the following reviewers for their work in referring manuscripts during 2018.

Salwa Salman Abed

Lorena Margo

Hasaballah Mohamed

Mustafa Kandem

Sabah Nasef

Selahatti

Maden

Gurdev Singh

Mustafa Avci

Tarek Sellami

Jorge Eliecer Hern

$\mathrm{Ndez} \mathrm{H}$

Ozgur Ege

Khaled Khalil Jaber

Hakem Ali

Fatma Ayca Cetinkaya

Hakan

Hossam A. Ghany

Sana Abughurra

Mohammad Javad Ebadi

Ankur Singh Bist

W. S. Amer

Ayyappan Govindasamy

Emilio Ram
Juid

Behnamf Razzaghf

Faizan Ahmed

Najah Ali Al-Ziadi

Ajaz Ahmad Dar

Muaadh Almahalebi

Rauhi I. Elkhatib

Kimuya M Alex

Fethi Bin Muhammad

Belgacem

Dr. Ramesh Prasad Aharwal

Shimaa A. Dessoky

Selami Ercan

Professor Zoran D Mitrovia

Mustafa Ozkan

Om Fatima Doaebil

Mustafa Turkyilmazoglu

Cesar Arturo Garza

Jeffrey Boyd

Murad Abuamr

Saratha Sathasivam

Shukur Neamah Al-Aeashi

Meltem Erden Ege

Maria Alessandra Ragusa
Vinodh Kumar Kumar

Chellamuthu

Fernane Khaireddine

Youssef El Foutayeni

Elena Corina Cipu

Shanmuga Sundaram O.V

Antonio Nicola Di Teodoro

Shalini Shekhawat

Mustafa Bilici

Quinn Jackson

Hasan

Inci Albayrak

Zhang Xi Wen

$\mathrm{K}$ Thirusangu

Erjola Cenaj

Muhammad Kamil Abidogun

Abdulrahman

Majid Erfanian

Fikri

Yunzhu Gao

Mustafa Gurbuz

Madan Mohan Panja

Francisco Bulnes

Ahmad Hamza Al Cheikha

Mehmet 